\title{
GENERALIZATION OF WAVEFORM COMMUNICATIONS: THE FOURIER ANALYZER APPROACH*
}

\author{
Géza Kolumbán, ${ }^{1}$ Francis C. M. Lau, ${ }^{2}$ and Chi K. Tse ${ }^{2}$
}

\begin{abstract}
In digital communications, fixed, chaotic, and random waveforms are used. Although the theory of fixed waveform communications is well established, its generalization to include chaotic and random carriers has not yet been carried out. The lack of an exact model prevents the optimization of chaotic and random communication systems.

Introducing the Fourier analyzer concepts, this paper develops a unified model which is equally valid for communications with fixed, chaotic and random carriers. It gives an exact measure for the a priori information which is exploited at the receiver to optimize the noise performance. The unified model may be used to determine the performance bounds of different waveform communication systems and offers a systematic approach for the development of optimum detector configurations. Using the model developed, a new classification of digital communication systems is proposed.
\end{abstract}

Key words: Waveform communications, subspace theory of detection, chaotic communications, Fourier analyzer concept, GML and ML decision rules.

\section{Introduction}

Digital communications with wideband signals are becoming one of the most important applications of radio communications [23]. Typical applications are the wireless local area network (WLAN), ultra-wideband radio (UWB), and sensor networks. It is not the thermal noise but the multipath propagation that limits the system performance in these, mostly indoor, applications; therefore, the transmitted signal must be a wideband signal.

* Received February 15, 2005; revised May 30, 2005; This research has been financed by the Hungarian Scientific Research Fund (OTKA) under Grant T-038083 and by the Hong Kong Polytechnic University under Grant G-T859.

${ }^{1}$ Department of Measurement and Information Systems, Budapest University of Technology and Economics, H-1521 Budapest, Hungary. E-mail: kolumban @mit.bme.hu

2 Department of Electronic and Information Engineering, The Hong Kong Polytechnic University, Hunghom, Hong Kong SAR, China. E-mail for Lau: encmlau@polyu.edu.hk; E-mail for Tse: encktse@polyu.edu.hk 
The digital information to be transmitted is mapped into fixed analog, mostly sinusoidal, waveforms in conventional digital communications. Basically, the conventional systems are narrowband, and the spectrum of the transmitted signal is spread by a pseudo-noise sequence [4] if a wideband transmitted signal is required.

An alternative solution is the application of a wideband carrier, where the digital information to be transmitted is directly mapped into a wideband signal. The carrier may be a fixed waveform (see impulse radio [23] as an example), a random signal (see the early attempt for implementing a wideband secure communication system with noise-like carrier [1] as an example), or a chaotic signal [3]. The common property of these solutions is that the carrier is a wideband signal; consequently, there is no need for an extra spectrum spreading sequence.

Because such UWB systems have not been used before, there are many open questions in this field, and the development of these systems is a great challenge for both system level engineers and circuit designers.

The theory of conventional telecommunications systems, where fixed waveforms are used as carriers, is very well established [20], [4]. In fixed waveform communications, if the same symbol is transmitted, then the same waveform is radiated.

In chaotic and random communications the transmitted waveforms are continuously varying, even if the same symbol is transmitted repeatedly. This unique property has a very strong influence on the applicable detector configurations. Frequently asked questions are: "How good or how bad is the theoretical noise performance of these systems compared to that of the conventional ones?" and "What is the theoretical noise performance bound of chaotic and random communication systems?"

To answer these questions the theory of waveform communications has to be extended to chaotic and random carriers. A unified theory and model must be established and developed, respectively, that (i) covers each kind of waveform communication system, (ii) may be used to determine the performance bounds of different waveform communication systems, and (iii) offers a systematic approach for the development of optimum detector configurations.

The a priori information available at the receiver is used in waveform communication to suppress channel noise and interference. As a rule of thumb we may say: the more the exploited a priori information, the better the system performance. The paper gives an exact measure for the a priori information. The measure proposed may be used in any kind of communications employing fixed, chaotic, and random carriers.

Section 2 extends the theory of communications with fixed waveforms to chaotic and random carriers by introducing the chaotic and random basis functions. It shows that in chaotic and random communications the basis functions are orthonormal only in mean. An undesirable consequence of this property is the estimation problem which corrupts the noise performance. 
In the channel model considered in Section 3, the transmitted signal is corrupted by an additive white Gaussian noise (AWGN) characterized by its power spectral density $N_{0} / 2$. The bandwidth of a noisy received signal is limited by an ideal bandpass channel filter. Recognizing that the detector observes the signal only over the signalling time period, a periodic signal is constructed. Using the Fourier series representation, a Hilbert space is defined; its dimension is determined by the product of bit duration and bandwidth of channel filter.

The detection is interpreted in such a way that using the a priori information at the receiver, subspaces are defined for each basis function in the observation space. These subspaces are observed by the detector, and an observation variable is generated for the decision circuit.

The last part of Section 3 gives a measure for the amount of a priori information that is equally valid for fixed, chaotic, and random basis functions.

Section 4 sets up the hierarchy of waveform communication systems and outlines the decision rules which may be used in the different classes of modulation schemes. The timing information is used to define a Hilbert space in which the different observation subspaces are defined by exploiting the a priori information available at the receiver. Then the observation variable is generated for the decision. The detector configuration to be built is developed from these decision rules, and references are given for each class of modulation schemes.

\section{Generalization of waveform communications}

Because only analog waveforms may be transmitted over a physical telecommunication channel and the data rate is given by the specification, the modulator of a digital telecommunication system maps the symbols to be transmitted into analog waveforms of finite duration. The waveform communication approach used here has nothing in common with analog modulation-techniques, where the amplitude, phase, or frequency of a sinusoidal signal is varied in accordance with the modulating signal. Instead, distinct waveforms, the carriers, are assigned to carry the different symbols. The carrier signals may be fixed, chaotic, or random waveforms.

To understand the unique feature of chaotic and random systems, consider a digital communication system which is suitable for the transmission of $m, m=$ $1,2, \ldots, M$ symbols. In the case of fixed carriers, if the same symbol is transmitted repeatedly, then the same waveform is generated in each bit duration by the modulator. Fixed carriers are used in conventional digital communications.

In the case of chaotic or random carriers, the transmitted waveform varies from symbol to symbol even if the same symbol is transmitted repeatedly. This unique property of chaotic and random carriers offers excellent multipath properties but deteriorates the noise performance. The source of noise performance degradation is the limited amount of a priori information on the transmitted signals. Because 
the transmitted waveforms are not fixed, only the statistical parameters of chaotic and random carriers are available.

In a digital modulator, the symbol $m$ is mapped into a signal vector $\mathbf{s}_{m}=\left[s_{m n}\right]$. In order to get the simplest mathematical model, the elements of the signal set are expressed as a linear combination of $N$ basis functions [4]

$$
s_{m}(t)=\sum_{n=1}^{N} s_{m n} g_{n}(t), \quad\left\{\begin{array}{l}
0 \leq t<T \\
m=1,2, \ldots, M \\
n=1,2, \ldots, N
\end{array}\right.
$$

where $N \leq M$. Each message is characterized by a distinct signal vector $\mathbf{s}_{m}=$ $\left[s_{m n}\right], m=1,2, \ldots, M$ and, according to (1), by a distinct waveform. The signalling time period $T$ of real-valued basis functions $g_{n}(t), n=1,2, \ldots, N$ is determined by the data rate. To avoid intersymbol interference (ISI), the value of the basis functions must be zero outside the signalling time interval.

To design a digital modulation scheme, either the elements $s_{m}(t)$ of the signal set or the basis functions $g_{n}(t)$ have to be chosen first. If the former are given, then the basis functions are determined by means of the Gram-Schmidt orthogonalization procedure [4]. If fixed carriers are used, then the basis functions are orthonormal:

$$
\int_{0}^{T} g_{i}(t) g_{n}(t) d t= \begin{cases}1, & \text { if } i=n \\ 0, & \text { otherwise }\end{cases}
$$

which means that each basis function carries unit energy and each pair of distinct basis functions is orthogonal to each other over the signalling time period $[0, T]$.

Each kind of digital receiver has more or less a priori information on the basis functions. This knowledge is exploited to suppress channel noise and interference. The more the a priori knowledge, the better the system performance.

The type of basis functions gives an upper bound on the a priori information that may be exploited by the receiver. Based on the basis functions, three classes of waveform communications are distinguished, namely communications with

- Fixed waveforms [4], [20], [22];

- Chaotic waveforms [3];

- Random waveforms [1].

The type of basis functions determines the noise and multipath performance of a telecommunication system. Before we develop the Fourier analyzer concept, the properties of basis functions are discussed in the next subsection.

\subsection{Types of basis functions}

In fixed waveform communications, the basis functions are fixed. Consequently, every time the same symbol is sent, the same waveform is transmitted. The basis 
functions and the elements of the signal set are exactly known. In built receivers the basis functions are recovered from the received signal (see the correlator receiver including a carrier recovery circuit) or stored at the receiver (see the matched filter approach) [20].

Note that the type of generator used to produce the fixed basis functions is irrelevant. Even a windowed part of a chaotic or random signal may be used as a basis function if it is stored at both the transmitter and receiver.

In chaotic communications, each basis function is the actual output of a chaotic signal generator. The chaotic signals are predictable only in the short run, because the chaotic systems have an extremely high sensitivity to the initial conditions and to the parameters of the chaotic attractor [19]. Because the shape of chaotic basis functions is not fixed, the radiated waveform varies even if the same symbol is transmitted repeatedly.

An important feature of chaotic communication systems is that the transmitted signal is never periodic. The chaotic signals are predictable only in the short run, so even theoretically only a limited amount of a priori information on basis functions is available.

In random communications, each basis function is the actual output of a random signal generator. The only difference between chaotic and random communication systems is that the random signals are not predictable even in the short run. Consequently, random communication systems are characterized by the minimum amount of a priori information available on the basis functions.

In the remainder of this work, the continuously varying property of chaotic and random basis functions is reflected by the upper index $q$,

$$
g_{n}^{q}(t), \quad q=1,2, \ldots,
$$

where $q$ identifies the basis function used to transmit the $q$ th element in a sequence of symbols. To get compact equations, $q$ will be suppressed except when we want to emphasize the time-varying property of basis functions.

Assume that symbol $m$ is transmitted $Q$ times. Using the notation we have just introduced, we get for fixed waveform communications

$$
g_{n}^{1}(t)=g_{n}^{2}(t)=\cdots=g_{n}^{Q}(t),
$$

whereas for chaotic and random communications each pair of basis functions is different:

$$
g_{n}^{1}(t) \neq g_{n}^{2}(t), \quad g_{n}^{1}(t) \neq g_{n}^{3}(t), \ldots, g_{n}^{l}(t) \neq g_{n}^{Q}(t), \ldots
$$

\subsection{Estimation problem}

At the receiver, the detector observes the received signal over the signalling time period called the bit duration $T$ and produces the observation variable. Because of the channel noise, it is a random variable characterized by its probability density function (pdf). Considering the noise performance, the most important parameters 
are the mean and standard deviation. The higher the variance of the observation variable, the worse the bit error rate (BER) [4]. Any effect that increases the variance of the observation variable corrupts the noise performance.

Random signals may be characterized only by their statistical parameters. Similarly, as the chaotic basis functions are not fixed waveforms, only their statistical parameters are available. To get these statistical parameters, the stochastic signal model has to be adopted to chaotic waveforms.

The chaotic stochastic signal model has been elaborated in [5], where the ensemble of sample functions is defined as the output of a chaotic attractor starting from all possible initial conditions.

Although both random signals and chaotic waveforms generated by different attractors $^{3}$ or starting from different initial conditions, of infinite length, are uncorrelated, and their corresponding average powers are constant, the random and chaotic basis functions are orthonormal over an interval of duration $T$ only in the mean:

$$
E\left[\int_{0}^{T} g_{i}^{q}(t) g_{n}^{q}(t) d t\right]=\left\{\begin{array}{ll}
1, & \text { if } i=n \\
0, & \text { otherwise }
\end{array},\right.
$$

where $E[\cdot]$ denotes the expectation operator. Note that from this respect there is no difference between the chaotic and random basis functions. In the remaining part of this subsection the properties of chaotic basis functions are discussed, but the results are also valid for the random basis functions.

The integral

$$
\int_{0}^{T} g_{i}^{q}(t) g_{n}^{q}(t) d t
$$

gives estimates of auto- $(i=n)$ and cross- $(i \neq n)$ correlations of basis functions [2]. These estimates calculated from sample functions of finite duration are random variables. Their variance increases the variance of the observation variable and, consequently, corrupts the noise performance. To get the best noise performance, the variances of auto- and cross-correlation estimations have to be kept zero.

For $i=n$, (4) estimates the energy of the $n$th basis function. Equation (4) means that if the chaotic signals are directly used as basis functions, then the energy used to transmit one symbol is not constant but varies from symbol to symbol. This property is called the autocorrelation estimation problem. The mean and standard deviation of estimation of $\int_{0}^{T}\left[g_{n}^{q}(t)\right]^{2} d t$ (i.e., the signal energy per symbol) versus estimation time (i.e., bit duration) are plotted in Figure 1, where the chaotic basis function was generated by a third-order analog phase locked loop (APLL) [12].

The effect of the cross-correlation estimation problem is shown in Figure 2,

${ }^{3}$ In this respect, attractors described by the same mathematical model but having different parameters are considered as different attractors. 


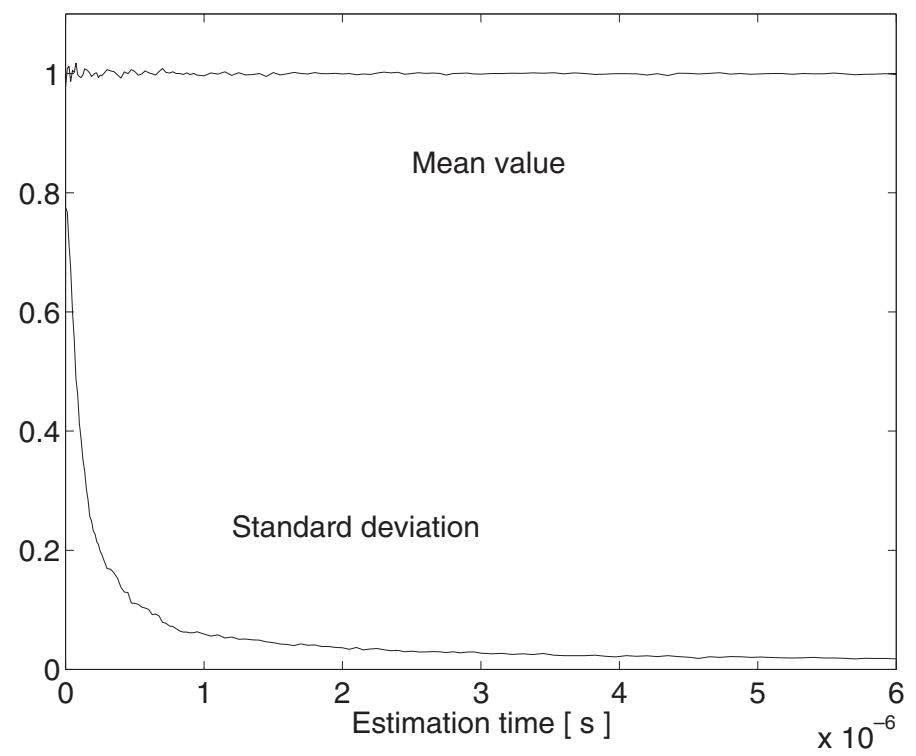

Figure 1. Autocorrelation estimation problem: mean and standard deviation of the estimation of energy carried by a chaotic basis function versus the estimation time.

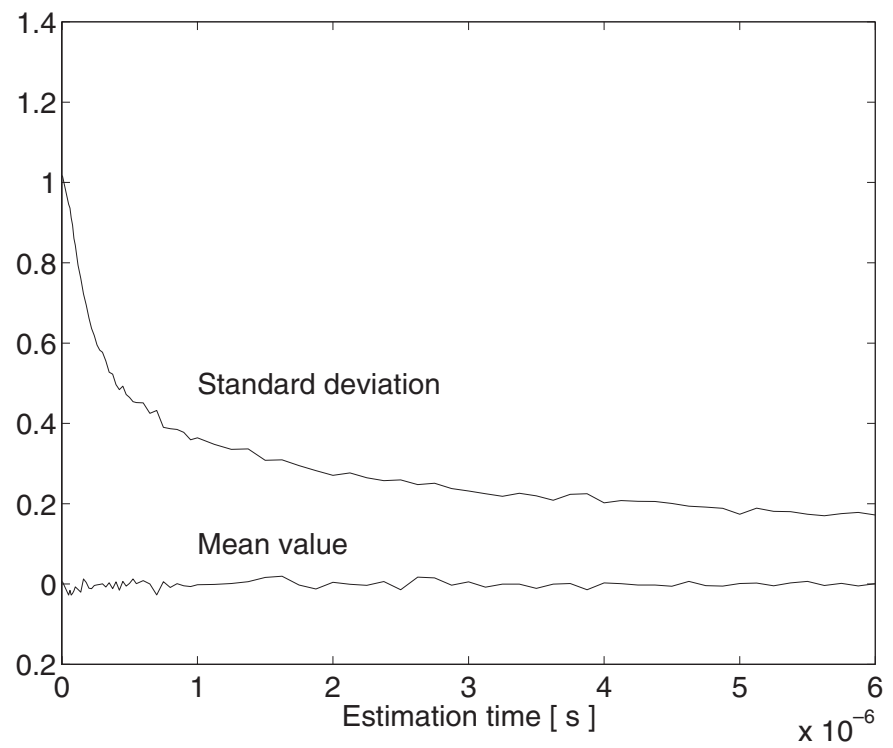

Figure 2. Cross-correlation estimation problem: mean and standard deviation of the estimation of cross-correlation of two chaotic basis functions versus the estimation time. 


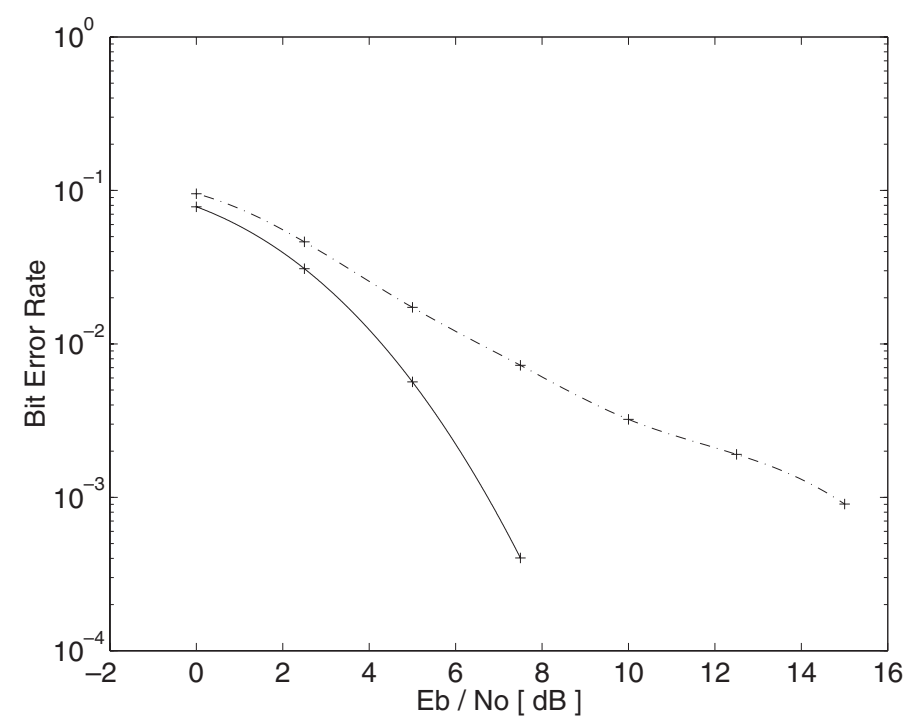

Figure 3. The noise performance of BCSK for basis functions with varying (dash-dotted curve) and constant (solid curve) energy.

where basis functions were generated by the same chaotic APLL. Due to the cross-correlation estimation problem, interference that corrupts the noise performance appears.

The standard deviation of both estimates is inversely proportional to the product of the estimation time (i.e., symbol duration $T$ ) and the equivalent statistical bandwidth of chaotic basis functions which is equal to the bandwidth $2 B$ of the bandpass transmitted signal.

To show the effect of the estimation problem, the noise performance of a binary antipodal chaos shift keying (BCSK) modulation scheme [7] has been determined for varying and constant energy per bit. Only one basis function is used in BCSK; consequently, only the autocorrelation estimation problem is present in this case. Figure 3 shows the manifestation of the estimation problem. The dash-dotted and solid curves give the noise performances as a function of energy per bit-to-noise spectral density ratio $E_{b} / N_{0}$ for the cases when the energy of basis function is varying or kept constant, respectively.

The estimation problem is present in every modulation scheme where the basis functions are orthonormal only in mean. It appears in both the chaotic and random modulation techniques. To solve the estimation problem there is no need to fix the shape of the basis function; it is enough to satisfy condition (2) of orthonormality for each sample of basis function.

An example is the frequency modulated differential chaos shift keying (FMDCSK) modulation scheme, where two basis functions are used. The constant 
energy and orthogonality of the basis functions are ensured by FM and the combination of the first two Walsh functions with the chaotic signals, respectively. The FM-DCSK basis functions are always orthonormal regardless of the shape of the chaotic signals [7]. This technique may also be applied to random signals to get orthonormal random basis functions.

\section{Signal model for detection}

In digital communications, the elements of the signal set carrying the symbols pass through a telecommunication channel in which they are corrupted by noise and may suffer from distortion, interference, and the multipath effect. Observing the corrupted and distorted received analog waveform for the interval of bit duration, the detector must decide which message has most likely been transmitted.

According to (1), the elements of the signal set are represented by basis functions that are known, or at least some of their characteristics are known at the receiver. This a priori knowledge is exploited to perform the detection and suppress channel noise and interference.

First a received signal space is defined in which each received signal such as transmitted signal, channel noise, and interference may be represented. Then the detection process is interpreted using the subspace detection theorem. In the simplest case, each basis function spans a subspace in the signal space. The detector maps the received signal into each subspace and generates an observation vector used later to perform the decision.

The subspace approach may be used either in the time domain [21] or in the frequency domain [17]. In the Fourier analyzer concept [11] used here, the signals are represented in the frequency domain.

This section shows how the Fourier analyzer concept may be used to develop a received signal space for the detection problem and gives a measure for the a priori information available for exploitation at the detector. This measure of a priori information determines how well a detector may separate the signal to be received from channel noise and interference; consequently, it determines the theoretical bound on noise performance.

\subsection{General block diagram of a receiver}

The general block diagram of a digital waveform communication receiver is shown in Figure 4. The transmitted signal $s_{m}(t)$ is corrupted by a signal $n(t)$ which may be the sample function of white Gaussian channel noise or an interference. The received signal is obtained as

$$
r_{m}(t)=s_{m}(t)+n(t) .
$$

To select the signal to be received, $r_{m}(t)$ is fed into a bandpass channel filter of 
460 Kolumbán, LAU, AND TSE

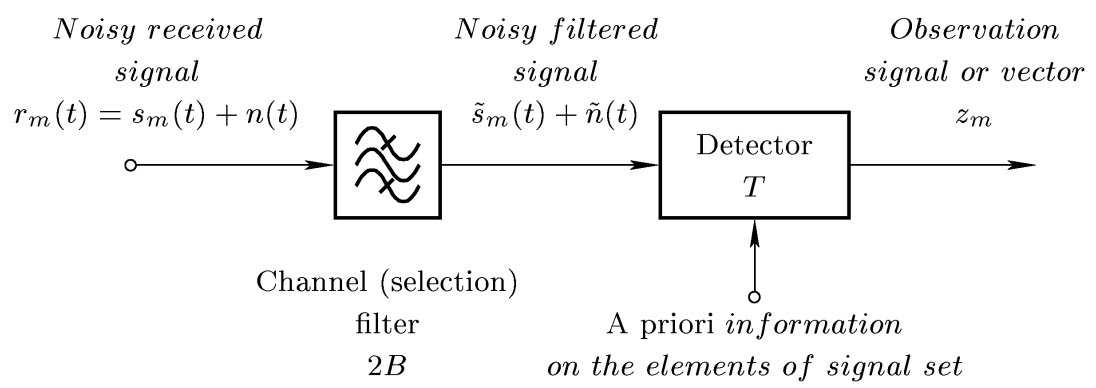

Figure 4. General block diagram of a digital waveform communication receiver.

RF bandwidth $2 B$. The detector observes the filtered received signal $\tilde{s}_{m}(t)+\tilde{n}(t)$ over the bit duration $T$ and generates the observation variable $z_{m}$; it may be either a random scalar number or a random vector.

The decision time instants, the bit duration $T$, and the RF bandwidth $2 B$ of transmitted signal $s_{m}(t)$ are always known at the receiver. Fortunately, these parameters are enough to apply the Fourier analyzer concept.

\subsection{Spectrum spreading}

The RF bandwidth $2 B$ of a telecommunication radio channel is limited; consequently, each element of signal set must be a bandpass signal. The duration of elements of a signal set is determined by the data rate.

Unfortunately, a signal of finite duration is not a bandpass signal [18]. To demonstrate this problem, assume that a bandpass signal with RF bandwidth of $2 B$, uniform spectrum, and infinitely long duration is generated. The basis function of duration $T$ is generated by windowing this signal.

The effect of windowing for $2 B T=4$ is shown in Figure 5, where the spectrum of the bandpass signal before and after windowing is plotted by dash-dotted and solid curves, respectively. The spectrum of the bandpass signal spreads after windowing, i.e., the basis functions of finite duration are not a bandpass signal any more. Their transmission over a bandpass channel may result in intersymbol interference [20].

If the data rate $1 / T$ is small enough compared to the RF bandwidth $2 B$ of the telecommunication channel, then the effect of spectrum spreading may be neglected, as shown in Figure 6. The figure plots the spectrum of a bandpass signal before (dash-dotted curve) and after (solid curve) windowing for $2 B T=34$. These parameters were used in a built WLAN FM-DCSK system operating in the 2.4-GHz ISM band [8].

In the remaining part of this paper the effect of spectrum spreading is neglected. 


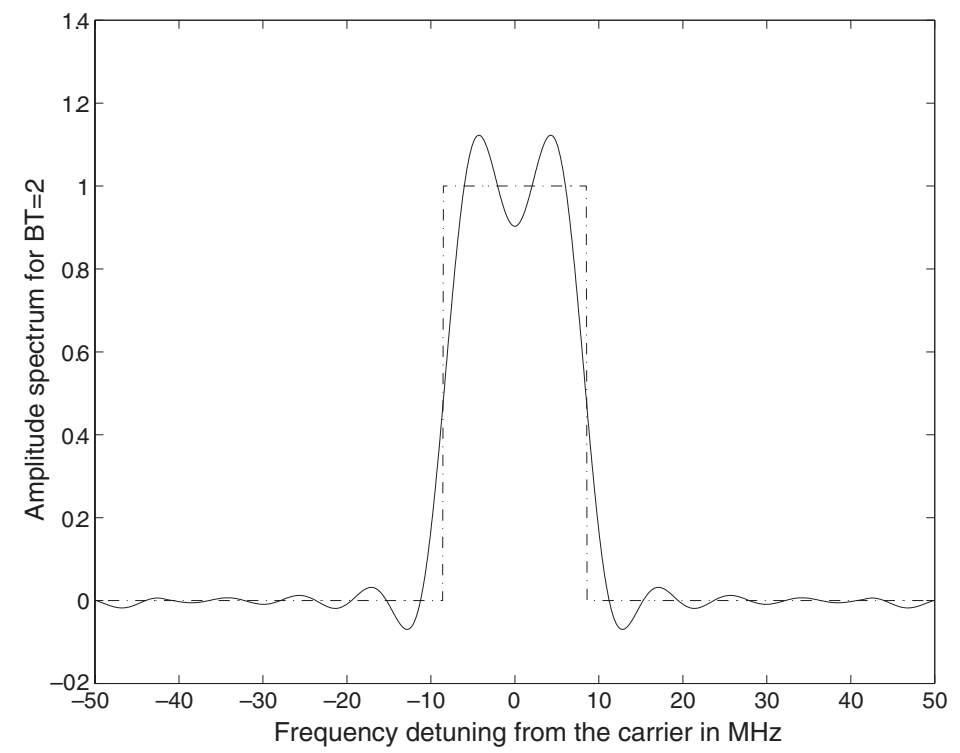

Figure 5. Amplitude spectra of an ideal bandpass signal before (dash-dotted curve) and after (solid curve) windowing for $2 B T=4$.

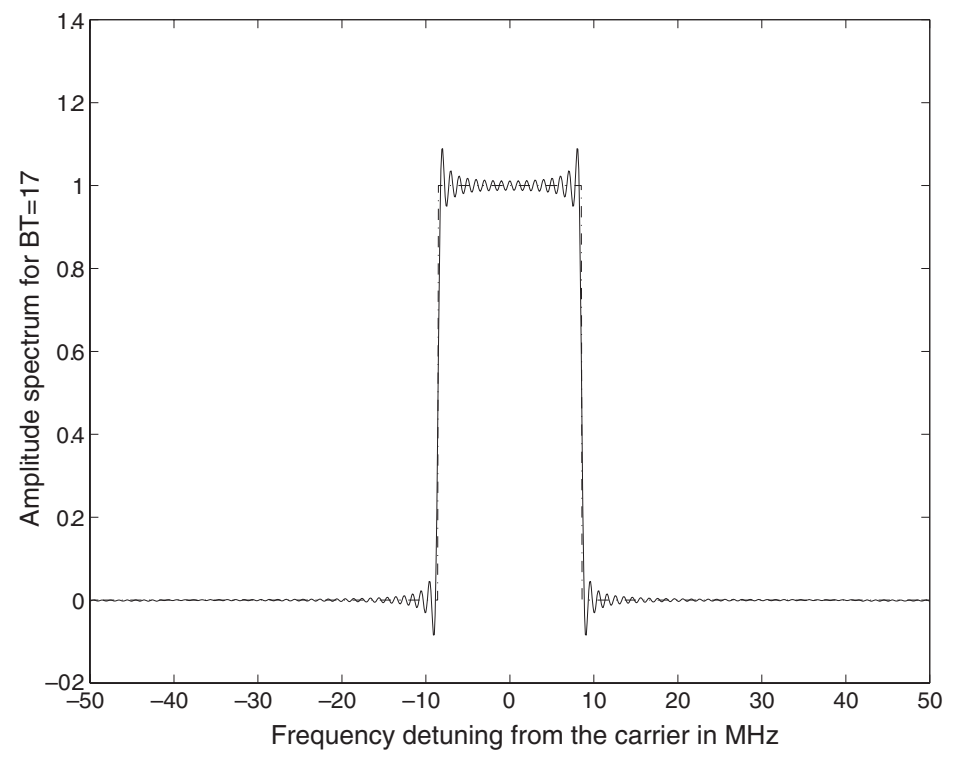

Figure 6. Spectrum spreading for $2 B T=34$. 


\subsection{Fourier analyzer concept}

To implement the subspace detection, first a received signal space has to be defined in which each signal appearing at the channel filter output may be represented.

The Fourier transform is widely used in electrical engineering to represent an arbitrary waveform in the frequency domain. However, the Fourier transform may not be used here because the dimension of its signal space goes to infinity.

Recall, the detector observes the channel filter output only over the bit duration. Outside the observation period, $\tilde{r}_{m}(t)$ may take any form. It may even be a periodic signal if it is identical to $\tilde{r}_{m}(t)$ over the bit duration. If the channel filter output is approximated by such a periodic signal, then the Fourier series representation can be used and a discrete spectrum is obtained. Because the detector input is a bandpass signal, the received signal space has a finite dimension.

To get a simple mathematical model, we interpret the received signal space as a Hilbert space [15] spanned by the harmonically related $\cos (\cdot)$ and $\sin (\cdot)$ functions. To distinguish the basis of the Hilbert space from the basis functions introduced by (1), let the former be called the Fourier base. In a Hilbert space an inner product and a norm have to be defined.

The inner product of two arbitrary waveforms $s_{1}(t)$ and $s_{2}(t)$ is defined as

$$
\left\langle s_{1}(t), s_{2}(t)\right\rangle=\int_{0}^{T} s_{1}(t) s_{2}(t) d t .
$$

Signals with zero inner product are called orthogonal signals.

Let the norm of a waveform $s(t)$ be defined as

$$
\|s(t)\|=\sqrt{\int_{0}^{T} s^{2}(t) d t}
$$

Note that the norm of a signal equals the energy carried.

\subsubsection{Fourier series representation}

Because a waveform communication receiver observes the received signal only over the bit duration, from the detection point of view a periodic signal of period $T$ may be constructed using

$$
s_{T, m}(t)=\left\{\begin{array}{ll}
s_{m}(t), & \text { for } 0 \leq t<T \\
s_{m}(t-C T), & \text { otherwise }
\end{array},\right.
$$

where $C$ is an arbitrary nonzero integer. 
The signal $s_{m}(t)$ may be represented by a Fourier series on the time interval $0 \leq t<T$

$$
\left.s_{m}(t)\right|_{0 \leq t<T}=s_{T, m}(t)=\sum_{k=K_{1}}^{K_{2}}\left[a_{k} \cos \left(k \frac{2 \pi}{T} t\right)+b_{k} \sin \left(k \frac{2 \pi}{T} t\right)\right] .
$$

Note that the fundamental period of the Fourier series representation is determined by the bit duration and has nothing to do with the center frequency of $s_{m}(t)$.

The Fourier series representation of $s_{T, m}(t)$ may be interpreted as a projection of $s_{T, m}(t)$ into a $2\left(K_{2}-K_{1}+1\right)$-dimensional Hilbert space, where the amplitudes of the signal components are equal to the following inner products weighted by $2 / T$ :

$$
\begin{aligned}
a_{k} & =\frac{2}{T} \int_{0}^{T} s_{T, m}(t) \cos \left(k \frac{2 \pi}{T} t\right) d t \\
& =\frac{2}{T}\left\langle s_{T, m}(t), \cos \left(k \frac{2 \pi}{T} t\right)\right\rangle \\
b_{k} & =\frac{2}{T} \int_{0}^{T} s_{T, m}(t) \sin \left(k \frac{2 \pi}{T} t\right) d t \\
& =\frac{2}{T}\left\langle s_{T, m}(t), \sin \left(k \frac{2 \pi}{T} t\right)\right\rangle .
\end{aligned}
$$

The Fourier base of the Hilbert space is the harmonically related

$$
\cos \left(k \frac{2 \pi}{T} t\right) \text { and } \sin \left(k \frac{2 \pi}{T} t\right)
$$

functions, where $K_{1} \leq k \leq K_{2}$. The dimension of the received signal space is determined in the next subsection.

Because the periodic signals have a discrete spectrum, the periodicity introduced by (5) makes the description of waveforms appearing at the detector input possible in a space of finite dimension. Note that the approximation introduced by $s_{T, m}(t)$ does not cause any distortion from the detection point of view as the periodic signal $s_{T, m}(t)$ coincides with waveform $s_{m}(t)$ on the interval $0 \leq t<T$, where the detector observes the signal.

Chaotic and random signals may also appear at the detector input. The Fourier series representation we have introduced remains valid for these signals, but the Fourier coefficients defined by (7) will be random variables. More details on the description of chaotic and random signals will be given in Section 3.3.3.

To complete the signal space model, the next subsection determines the relationship between the dimension of the Hilbert space and the parameters of the waveform communication receiver. 


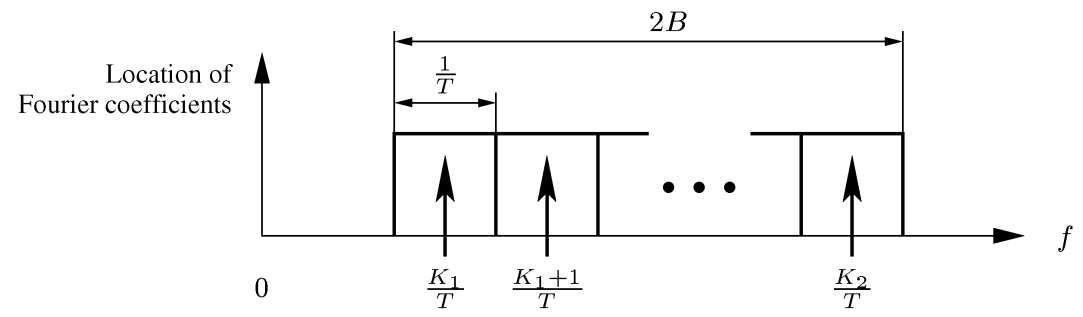

Figure 7. Determination of the dimension of received signal space: location of Fourier coefficients of detector input in the frequency domain.

\subsubsection{Signal dimension}

Suppose that the effect of spectrum spreading may be neglected, i.e., $2 B T>>1$. As shown in Figure 6, the detector input is a bandpass signal, the bandwidth of which is determined by the channel filter. Consider an ideal bandpass channel filter with bandwidth $2 B$ and center frequency $f_{0}$. In a well-designed receiver the channel filter is matched to the transmitted signal; consequently, the channel filter passes the elements of the signal set without distortion:

$$
\tilde{s}_{m}(t)=s_{m}(t) \text {. }
$$

Let $S_{m}(\omega), m=1,2, \ldots, M$, denote the Fourier transform of the elements of signal set. As $\tilde{s}_{m}(t)=s_{m}(t)$, substituting (5) into (7) and using the definition of the Fourier transform, we get

$$
a_{k}=\frac{2}{T} \operatorname{Re}\left[S_{m}\left(k \frac{2 \pi}{T}\right)\right]
$$

and

$$
b_{k}=-\frac{2}{T} \operatorname{Im}\left[S_{m}\left(k \frac{2 \pi}{T}\right)\right] .
$$

Because $s_{m}(t)$ is a bandpass signal, only a finite number of Fourier coefficients differs from zero.

Figure 7 shows the location of the Fourier coefficients of the detector input by arrows in the frequency domain. According to (6), the detector input has a discrete spectrum, and the distance between two adjacent spectral components is equal to the data rate $1 / T$. The bandwidth of the detector input is limited by the channel filter, and this ideal bandpass filter suppresses each spectral component lying outside the frequency range $\left(2 K_{1}-1\right) / 2 T \leq f \leq\left(2 K_{2}+1\right) / 2 T$. Note that only a finite number of Fourier coefficients differs from zero.

By definition, the signal dimension gives the number of harmonically related $\sin (\cdot)$ and $\cos (\cdot)$ functions along which the receiver collects information on the received signal. In other words, the signal dimension gives the dimension of the Hilbert space spanned by the Fourier base which is required to represent any 
signal appearing at the detector input over the observation time interval. From Figure 7 and (6), the signal dimension is obtained as

$$
S_{D}=2\left(K_{2}-K_{1}+1\right)=4 B T,
$$

where

$$
K_{1}=\frac{2\left(f_{0}-B\right) T+1}{2}=\frac{4 f_{0} T-S_{D}+2}{4}
$$

and

$$
K_{2}=\frac{2\left(f_{0}+B\right) T-1}{2}=\frac{4 f_{0} T+S_{D}-2}{4} .
$$

Note that the signal dimension is independent of the center frequency of the telecommunication channel. It is determined by the product of channel bandwidth and bit duration.

\subsubsection{Properties of basis functions}

Every signal, either deterministic, chaotic, or random, appearing at the detector input and having a duration $T$ may be represented in the received signal space spanned by the Fourier base. This representation is not an approximation but an exact description of any signal over the signalling time interval.

Only a subspace of the received signal space is required to represent the elements of the signal set used to transmit the information. To minimize the effect of channel noise and interference on the observation variable, the detector observes only that subspace where the energy of the transmitted waveform is greater than zero.

The basis functions in (1) have been introduced to describe the elements of a signal set with a minimum number of waveforms. The subspaces where the energy of the transmitted signal is greater than zero are assigned by the basis functions; each subspace belongs to a basis function. The union of these subspaces constitutes the observation space. To get the subspaces of each basis function, they have to be represented in the $S_{D}$-dimensional Hilbert space, that is, in the received signal space.

Using the technique introduced in the previous paragraph, the basis functions are obtained in the received signal space as

$$
\left.g_{n}^{q}(t)\right|_{0 \leq t<T}=g_{T, n}^{q}(t)=\sum_{k=K_{1}}^{K_{2}}\left[\alpha_{n k}^{q} \cos \left(k \frac{2 \pi}{T} t\right)+\beta_{n k}^{q} \sin \left(k \frac{2 \pi}{T} t\right)\right],
$$

where the Fourier coefficients $\alpha_{n k}^{q}$ and $\beta_{n k}^{q}$ are obtained from (7) and the constants $K_{1}$ and $K_{2}$ are given by (8) and (9), respectively.

The orthogonality of the basis functions makes them distinguishable in the received signal space. Only a subset of the Fourier coefficients in (10) differs 
from zero. The subspace defined by these nonzero Fourier coefficients is referred to as the subspace of the basis function $g_{n}(t)$.

The upper index $q$ in (10) indicates that the basis functions are not necessarily constant. In fixed waveform communication the basis functions are fixed and $q$ is dropped. Substituting (10) into (2), the orthonormality of the fixed basis functions is obtained as a function of the Fourier coefficients

$$
\sum_{k=K_{1}}^{K_{2}}\left(\alpha_{i k} \alpha_{n k}+\beta_{i k} \beta_{n k}\right)=\frac{T}{2} \delta_{i n} .
$$

In the case of chaotic and random basis functions, the Fourier coefficients $\alpha_{n k}^{q}$ and $\beta_{n k}^{q}$ in (10) become random variables. Substituting (10) into (3), we have

$$
E\left[\sum_{k=K_{1}}^{K_{2}}\left(\alpha_{i k}^{q} \alpha_{n k}^{q}+\beta_{i k}^{q} \beta_{n k}^{q}\right)\right]=\frac{T}{2} \delta_{i n} .
$$

\subsection{Measure of a priori information}

In waveform communications, either fixed, chaotic, or random, two kinds of information are available at the receiver, the must know and a priori information.

Without the must know information, the detection cannot be performed. The must know information includes the timing information expressed by the bit duration $T$ here, and the spectral properties of the transmitted signal characterized by the RF bandwidth $2 B$ in the Fourier analyzer concept.

The a priori information is exploited by the detection algorithm to suppress the effect of channel noise and interference. The detection may be performed even if only a very limited amount of a priori information is available at the receiver, but the less the a priori information, the worse the BER.

Starting from the recognition that the detector observes the received signal only over the signalling time interval $0 \leq t<T$, the Fourier series representation has been used to describe each waveform in a finite-dimensional Hilbert space of harmonically related $\sin (\cdot)$ and $\cos (\cdot)$ functions. The importance of the received signal space is that any signal appearing at the detector input may be represented in this Hilbert space over the bit duration. In this sense the received signal space is a complete space. The fundamental period of the sinusoidal Fourier base of the received signal space equals the bit duration.

Because of the channel filter, the detector input is a bandpass signal. The bandwidth of the channel filter matched to the elements of the signal set determines the dimension $S_{D}$ of the Hilbert space.

A subspace of the $S_{D}$-dimensional received signal space is observed by the detector, and the dimension of this observation space is determined by the number of nonzero Fourier coefficients of the basis functions. Note that only the basis functions and, consequently, the elements of the signal set may be represented in 
the observation space. The observation space is not suitable for the representation of an arbitrary signal such as channel noise or interference.

In conventional telecommunications, coherent, differentially coherent, and noncoherent receivers are distinguished [22], [20]. The noncoherent detection is considered as the detection of signals with unknown phase [4].

The conventional classification of modulation techniques cannot be extended to generalized waveform communications, because in the latter the basis functions

- may have any shape, consequently, many times they have no phase;

- may vary from symbol to symbol even if the same symbol is transmitted repeatedly.

Equation (1) shows that the transmitter radiates signal energy only in the subspaces assigned by the basis functions. In the complementary subspace only noise and interference are received. The detector suppresses these unwanted signals in such a way that it observes only the subspaces assigned by the basis functions, i.e., where the transmitted signal energy is greater than zero. This technique is referred to as the subspace detection theorem.

If the Fourier components are exactly known at the receiver, then the subspaces defined at the detector using the a priori information are identical with the subspaces assigned by each transmitted basis function, and the maximum amount of noise and interference is suppressed. If the a priori information is not enough to define without any error the subspace of each basis function, then the observation space differs from the one assigned by the basis functions at the transmitter. Because of the error, signal energy is lost and extra channel noise and interference is accommodated in the observation variable. Consequently, the system performance is corrupted.

To classify the waveform modulation techniques, a mathematical measure of a priori information should be defined that

- is independent of the implementation of the detection algorithm;

- may be used for any kind of basis functions.

Equation (10) shows that the construction of the observation subspace of the $n$th basis function requires the regeneration of the Fourier base and the knowledge of Fourier coefficients $\alpha_{n k}$ and $\beta_{n k}$. The Fourier base is regenerated from the timing information that is always available. The implemented demodulators differ in how well the Fourier coefficients are known at the receiver.

In the Fourier analyzer concept, the measure of available a priori information relates to how precisely the Fourier coefficients of the basis functions are known at the receiver.

For example, in a conventional coherent frequency-shift keying (FSK) receiver the Fourier coefficients are exactly known. However, an FSK signal may also be detected by a noncoherent receiver, where only the signalling frequencies are known at the receiver. In the Fourier analyzer concept it means that the exact val- 
ues of $\alpha_{n k}$ and $\beta_{n k}$ are not available, but the values of $k$ for which $\sqrt{\alpha_{n k}^{2}+\beta_{n k}^{2}}>$ $0, n=1,2, \ldots, N$ are known. Then the signalling frequencies are obtained as $k / T$.

The next section classifies the waveform communication systems using this measure of a priori information.

\section{Hierarchy of modulation techniques}

Section 2 has distinguished communication with fixed, chaotic, and random basis functions. The type of basis function has the strongest effect on the implementation and performance of a communication system.

The classification of modulation schemes in the theory of conventional telecommunication systems is strongly influenced by implementation issues. For example, coherent communication is frequently considered as reception by a correlation or a matched filter receiver.

However, the modulation scheme and implementation of a receiver are two completely independent issues. For example, an FSK signal may be demodulated by either a coherent correlation or a noncoherent receiver. The separation of waveforms in a signal set and the a priori information on the Fourier coefficients of basis functions determine the performance bound of a modulation scheme. The implementation of a detector determines to what extent this information is exploited. A noncoherent receiver is frequently used even if enough a priori information is available to design a coherent one in order to reduce cost or power consumption.

Section 3.4 has shown that the accuracy with which the Fourier series coefficients in (10) are known equals the amount of a priori information available at the receiver. This parameter relates to how precisely the subspaces of each basis function are known at the receiver. Using this measure, a new classification of modulation schemes is introduced here that is independent of receiver implementation and the type of basis function.

Electronic circuits process the amplitude and phase of bandpass signals. To follow this terminology, let the complex Fourier coefficients of basis functions, given by

$$
\gamma_{n k}^{q}=\frac{1}{2}\left(\alpha_{n k}^{q}-j \beta_{n k}^{q}\right) \quad \text { and } \quad \gamma_{n(-k)}^{q}=\frac{1}{2}\left(\alpha_{n k}^{q}+j \beta_{n k}^{q}\right),
$$

be considered in the classification of modulation techniques.

\subsection{Pure coherent modulation technique}

Let an $S_{D}$-dimensional received signal space be considered, where $K_{1} \leq k \leq K_{2}$ and $S_{D}=2\left(K_{2}-K_{1}+1\right)=4 B T$. In a pure coherent modulation scheme, all complex Fourier coefficients $\gamma_{n k}^{q}$ are exactly known. 
In fixed waveform communications, the complex Fourier coefficients $\gamma_{n k}$ are constant, and they are recovered by a carrier recovery circuit (see coherent correlation receiver as an example) or stored as the impulse response of a matched filter.

In chaotic or random waveform communications, the Fourier coefficients vary from symbol to symbol; consequently, the matched filter approach cannot be used. Unfortunately, a robust solution to the recovery of chaotic or random basis functions from the received noisy and distorted signal has not yet been published. This is why the coherent modulation technique cannot be used in the implemented chaotic and random communication systems.

In a pure coherent receiver, the Fourier coefficients are exactly known, and the maximum likelihood (ML) [20], [4] decision rule is applied which ensures the best noise performance. For the development of the ML decision rule and coherent receiver configuration, refer to [16].

\subsection{Quasi coherent modulation technique}

The pure coherent modulation technique can never be implemented because some noise (in the case of carrier recovery) and error in reproducing the Fourier coefficients are always present. If the noise and error are small enough, then the modulation scheme is referred to as a quasi coherent technique, and the ML decision rule is still applied. The noise and error cause a loss in a priori information and result in a performance degradation.

The quasi coherent modulation technique is used until the loss in BER caused by the noise and error is less than the loss caused by the application of the generalized maximum likelihood (GML) [17] decision rule.

\subsection{Optimum noncoherent modulation technique}

In the optimum noncoherent modulation technique there is a further loss in a priori information as only the absolute values of complex Fourier coefficients $\gamma_{n k}$ are known. This technique may be used only in fixed waveform communication. An example of this modulation technique is the optimum differentially coherent detection of a binary differential phase-shift keying (DPSK) signal (see Fig. 8.30 in [4]).

Because the phases of the complex Fourier coefficients are not known, the a priori information available at the receiver includes only the absolute values of the complex Fourier coefficients and their frequencies. The former information is exploited to optimize the signal-to-noise ratio (SNR) in the observation variable, the latter one is used to define the observation space. The technique used to maximize the SNR in the observation variable is referred to as the GML decision rule [17]. 


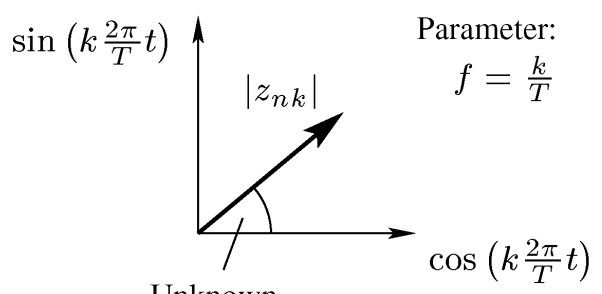

phase angle

Figure 8. Optimum noncoherent modulation: effect of unknown phase of complex Fourier coefficients. The observation space is defined by the frequencies $k / T$ where signal energy is transmitted.

To highlight the effect of loss in a priori information on noise performance, consider the observation of $k / T$-frequency components of the $n$th basis function. Because the observation space is defined by the frequencies along which signal energy is transmitted, the detector must observe both the $\cos (k 2 \pi t / T)$ and $\sin (k 2 \pi t / T)$ directions to collect all signal components, as shown in Figure 8, where $z_{n k}$ is the $k / T$-frequency component of the observation signal.

Suppose that the actual value of the phase angle of $\gamma_{n k}$ is zero, i.e., $\gamma_{n k}$ coincides with the direction of $\cos (k 2 \pi t / T)$. In this case only noise appears along the direction $\sin (k 2 \pi t / T)$. However, as the phase of the complex Fourier coefficients is not known at the receiver, both directions have to be observed and the noise appearing along the direction of $\sin (k 2 \pi t / T)$ is also accommodated in the observation variable, increasing its variance, i.e., deteriorating the noise performance. The larger the variance, the worse the noise performance.

\subsection{Averaged optimum noncoherent modulation technique}

The idea of optimum noncoherent modulation technique is extended to chaotic and random waveforms by introducing the idea of an averaged optimum noncoherent modulation technique. The basis functions vary from symbol to symbol in this case, so only the averages

$$
E\left[\left|\gamma_{n k}^{q}\right|\right]
$$

of the absolute values of the complex Fourier coefficients are known.

The detection algorithm is very similar to that one discussed in Section 4.3. The difference is that here the averages of $\left|\gamma_{n k}^{q}\right|, n=1,2, \ldots, N$ have to be used as weights in generating the observation variable. This approach is referred to as the GML detection rule with averaged weights and is discussed in [10]. The fact that only an averaged value of weights is known causes a further loss in a priori information and results in a further degradation in noise performance.

The GML decision rule with averaged weights was applied to FM-DCSK in [10] in order to optimize its noise performance. 


\subsection{Noncoherent modulation technique}

The least a priori information is exploited in the noncoherent modulation technique. The detection algorithm is very similar to the one which was discussed in Section 4.3. The difference is that here the weights have only two distinct values. Because of the fixed weights, this approach may be equally used for communications with fixed, chaotic, and random basis functions. This technique is referred to as the GML detection rule with binary weights.

The subspaces of each basis function are defined by a set of constants $W_{n k}$ which are also used as weights in generating the observation variable. The weights have only two values, their value is 1 and 0 if the signal component is and is not, respectively, transmitted:

$$
W_{n k}= \begin{cases}1, & \text { if } E\left[\left(\alpha_{n k}^{q}\right)^{2}+\left(\beta_{n k}^{q}\right)^{2}\right]>0 \\ 0, & \text { if } E\left[\left(\alpha_{n k}^{q}\right)^{2}+\left(\beta_{n k}^{q}\right)^{2}\right]=0\end{cases}
$$

Because identical weights are used for each component of the observation variable, the noise contribution of a component is not controlled by its SNR. This causes a further loss in a priori information and results in the worst noise performance. Even more, the noise performance depends on the signal dimension. The higher the signal dimension, the worse the noise performance [17], [6].

Although the noncoherent modulation technique offers the worst noise performance in an AWGN channel, it is very robust against the channel distortion. Examples of fixed waveform communications are the autocorrelation reception of DPSK signals [17] and the autocorrelation reception of a transmitted reference (TR) impulse radio system [23] developed for UWB radio applications.

In chaotic communications two kinds of noncoherent receivers have been developed, the autocorrelation receiver [13] and the energy detector [9]. Both of them are suitable for the reception of DCSK and FM-DCSK signals.

\subsection{Closing remarks}

Note that in going from the pure coherent modulation technique to the noncoherent one there is a continuous loss in a priori information. The ability of a receiver to suppress noise and interference depends on the amount of a priori information available and exploited by the detector. Consequently, if only the noise performance and interference rejection is considered, then the pure coherent and noncoherent modulation techniques offer the best and worst, respectively, system performance. 


\section{Conclusions}

Exploiting the Fourier analyzer approach, a new model has been developed for waveform communications. The new model is valid for any kind of carriers; they may be fixed, chaotic, or random waveforms.

The detector of a digital communication system observes the received waveform only over the signalling time period. Consequently, a periodic signal may be constructed that is identical to the received one over the signalling time period. The periodic signal is represented by a Fourier series, i.e., it has a discrete spectrum. The Fourier base of the signal space is determined by the timing information, which is always available at the receiver. The fundamental frequency of the Fourier base equals the bit duration.

The signal space constitutes a Hilbert space. Its dimension, referred to as the signal dimension, is determined by the product of bit duration and bandwidth of the channel filter. The subspaces of each basis function are defined by the Fourier coefficients.

Unfortunately, the Fourier coefficients are not always exactly known at the receiver. The accuracy with which the Fourier coefficients are known determines the type of receiver.

The question in the design of a detector is how to distinguish the signal from noise, how to suppress the noise but collect the signal components. The available a priori information is used to perform this duty in such a way that the observation subspaces are defined by means of this information. The more accurate the a priori information, the less sensitive the detector is to channel noise and interference.

An exact measure of a priori information available at the receiver has been introduced here and, based on this measure, a new classification of waveform communication techniques has been proposed. Compared to the fixed waveform communication systems, the chaotic ones suffer from a theoretical loss in a priori information and there is a further information loss in random communications. Consequently, the noise performance of chaotic communication systems over an AWGN channel is worse than that of fixed waveform communication systems, and the random systems offer the worst noise performance.

Until this time the predictability of chaotic signals in the short run [19] has not yet been exploited. The question is whether this predictability in the time domain could be exploited in real applications to improve the noise performance of a chaotic communication system. This research field is referred to as noise cleaning [14].

A computer simulation of noise cleaning has shown that the noise performance of a chaotic DCSK system may be slightly improved (less than 1-dB improvement in $E_{b} / N_{0}$ ) for the region where the channel noise is relatively low [24]. Unfortunately, the noise cleaning algorithms have a threshold effect and the noise performance becomes slightly worse in that region of $E_{b} / N_{0}$ which has to be considered in real radio applications. Because of the unsatisfactory improvement 
in noise performance and computationally intensive algorithm required by noise cleaning, application of noise cleaning to communications has been abandoned.

\section{References}

[1] B. L. Basore, Noise-like signals and their detection by correlation, Ph.D. thesis, MIT, Cambridge, MA, 1952.

[2] J. S. Bendat and A. G. Piersol, Measurement and Analysis of Random Data, New York: John Wiley \& Sons, 1966.

[3] M. Hasler, G. Mazzini, M. Ogorzalek, R. Rovatti, and G. Setti, Special issue on applications of nonlinear dynamics to electronic and information engineering, Proceedings of the IEEE, vol. 90, May 2002.

[4] S. Haykin, Communication Systems, New York: John Wiley \& Sons, 3rd ed., 1994.

[5] G. Kis and G. Kolumbán, Constraints on chaotic oscillators intended for communications applications, in Proc. NOLTA'98, (Crans-Montana, Switzerland), pp. 883-886, September 14$17,1998$.

[6] G. Kolumbán, Theoretical noise performance of correlator-based chaotic communications schemes, IEEE Trans. Circuits and Systems-Part I: Fundamental Theory and Application, vol. 47, pp. 1692-1701, December 2000.

[7] G. Kolumbán and M. P. Kennedy, The role of synchronization in digital communication using chaos-Part III: Performance bounds, IEEE Trans. Circuits and Systems-Part I: Fundamental Theory and Application, vol. 47, pp. 1673-1683, December 2000.

[8] G. Kolumbán, M. P. Kennedy, Z. Jákó, and G. Kis, Chaotic communications with correlator receiver: Theory and performance limit, Proceedings of the IEEE, vol. 90, pp. 711-732, May 2002.

[9] G. Kolumbán and G. Kis, Reception of $M$-ary FM-DCSK signals by energy detector, in Proc. NDES'03, (Scuol, Switzerland), pp. 133-136, May 18-22, 2003.

[10] G. Kolumbán, G. Kis, F. C. M. Lau, and C. K. Tse, Optimum noncoherent FM-DCSK detector: Application of chaotic GML decision rule, in Proc. IEEE-ISCAS'04, (Vancouver, Canada), pp. 597-600, May 23-26, 2004.

[11] G. Kolumbán, F. C. M. Lau, and M. Small, A new description of chaotic waveform communications: The Fourier analyzer approach, in Proc. ECCTD'03, vol. III, (Cracow, Poland), pp. 241244, September 1-4, 2003.

[12] G. Kolumbán and B. Vizvári, Nonlinear dynamics and chaotic behaviour of the analog phaselocked loop, in Proc. NDES'95, (Dublin, Ireland), pp. 99-102, July 1995.

[13] G. Kolumbán, B. Vizvári, W. Schwarz, and A. Abel, Differential chaos shift keying: A robust coding for chaos communication, in Proc. NDES'96, (Seville, Spain), pp. 87-92, June 27-28, 1996.

[14] E. J. Kostelich and T. Schreiber, Noise reduction in chaotic time series data: A survey of common methods, Physical Review E, vol. 48, p. 1752, 1993.

[15] E. Kreyszig, Advanced Engineering Mathematics, New York: Wiley, 1999.

[16] F. C. M. Lau and G. Kolumbán, Performance limit of chaotic digital waveform communication systems: Approach of maximizing a posteriori probability, Circuits, Systems and Signal Processing, vol. 24, pp. 639-655, 2005.

[17] Y. B. Okunev, Phase and Phase-Difference Modulation in Digital Communications, Boston: Artech House, 1997.

[18] A. V. Oppenheim, A. S. Willsky, and I. T. Young, Signals and Systems, Englewood Cliffs: Prentice-Hall, 1983.

[19] T. S. Parker and L. O. Chua, Practical Numerical Algorithms for Chaotic Systems, New York: Springer-Verlag, 1989. 


\section{Kolumbán, LAU, AND TSE}

[20] J. G. Proakis, Digital Communications, Singapore: McGraw-Hill, 1995.

[21] A. B. Salberg and A. Hanssen, Stochastic multipulse-PAM: A subspace modulation technique with diversity, Signal Processing, vol. 83, pp. 2559-2577, December 2003.

[22] M. K. Simon, S. H. Hinedi, and W. C. Lindsey, Digital Communication Techniques: Signal Design and Detection, Englewood Cliffs, New Jersey: PTR Prentice Hall, 1995.

[23] K. Siwiak and D. McKeown, Ultra-Wideband Radio Technology, Chichester, UK: Wiley, 2004.

[24] Z. Jákó and G. Kis, Application of noise reduction to chaotic communications: A case study, IEEE Trans. Circuits and Systems-Part I: Fundamental Theory and Application, vol. 47, pp. 1720-1725, December 2000. 